\title{
Image Despeckling Based on Improved LMMSE Wavelet Shrinkage
}

\author{
Yanqiu Cui ${ }^{1,2}$ \\ ${ }^{1}$ Faculty of Electronic Information and Electrical \\ Engineering, \\ Dalian University of Technology, \\ ${ }^{2}$ College of Information\& Communication Engineering \\ Dalian Nationalities University \\ Dalian, China \\ cyq@dlnu.edu.cn
}

\author{
Min Han \\ Faculty of Electronic Information and Electrical \\ Engineering, \\ Dalian University of Technology, \\ Dalian, China \\ minhan@dlut.edu.cn
}

\begin{abstract}
The automatic interpretation of SAR images is often extremely difficult due to speckle, a signal dependent noise, which is inherent of all active coherent imaging systems. Thus, despeckling has become a crucially important issue in SAR image processing. Wavelet theory provides a powerful tool for detecting image feature at different scales. Wavelet-based algorithms have been widely used to reduce speckle noise. In this paper, an adaptive despeckling method for synthetic aperture radar (SAR) images is proposed based on wavelet shrinkage. It follows the framework of the linear minimum mean square error (LMMSE) filter in the wavelet domain proposed for speckle suppression, but improves the parameter estimation method by taking into account the distribution property of wavelet coefficients based on the bilateral kernel regression. An improved adaptive shrinkage function is obtained and each coefficient is decided separately. Simulation results for the simulated SAR images demonstrate the proposed modified method outperforms some representative SAR despeckling methods when the noise is not serious.
\end{abstract}

Keywords-synthetic aperture radar (SAR); speckle; wavelet transform; linear minimum mean square error (LMMSE); kernel regression

\section{INTRODUCTION}

Synthetic aperture radar (SAR) has the capability of the all-day, all-weather acquisition. Therefore, SAR images provide useful information for many applications, such as reconnaissance, surveillance, and targeting. However, the automatic interpretation of SAR images is often extremely difficult due to speckle, a signal dependent noise, which is inherent of all active coherent imaging systems. Thus, despeckling has become a crucially important issue in SAR image processing.

The increasing number of algorithms has been developed to suppress speckle noise. Usually, the speckle is partially suppressed already during the image formation process by multilook processing. But multilook averaging reduces the standard deviation of speckle but it also deteriorates the spatial resolution. Other traditional approaches are spatial filtering, for example the Fuan filter [1], and their variations such as the enhanced Fuan filter. The performance of these filters depends heavily on the choice of the local window, and exhibit limitations in preserving the detail in weakly textured areas.
To overcome these disadvantages, wavelet-based algorithms [2] have been widely used to reduce speckle noise. Wavelet theory provides a powerful tool for detecting image feature at different scales. Due to this property, the despeckling approaches based on wavelet transform can well preserve details of the original image. Speckle in SAR images is multiplicative, whereas most existing wavelet denoising algorithms are developed for additive noise. To take advantage of the available algorithms based on wavelet transform, the log-transform is applied to SAR image to convert the multiplicative noise to additive noise before performing wavelet denoising [3, 4]. After wavelet denoising on log-transformed image, an exponential operation is employed to convert the image back to a non-logarithmic format. The major disadvantage of such approaches is that the backscatter mean is not preserved in homogeneous areas when the image is converted back to a non-logarithmic format after denoising. Furthermore, signal variations are damped by the logarithm, resulting in an unlikely "flatness" after despeckling.

To eliminate the impact of log-transform to denoising performance, some algorithms without performing the logarithmic transform have been proposed. Xie et al. propose a low-complexity wavelet denoising process based on the minimum mean square error (MMSE) estimation [5]. But the discrete wavelet transform (DWT) is applied during image denoising. The DWT is critically subsampled and time variant, which affects the performance of the despeckling. Alternative approaches have been proposed that are based on the stationary wavelet transform (SWT), which is a time-invariant transform. Foucher et al. propose a SWT despeckling algorithm using the maximum a posteriori (MAP) criterion [6], in which the Pearson distribution is used to model the probability density function (pdf) of wavelet coefficients. Although this algorithm has good performance, the high computational complexity of the Pearson distribution makes this approach rarely used in practice. To simplify calculations, Argenti et al. introduce a local linear MMSE (LMMSE) filter [7]. Dai et al. present a despeckling method based on the mixture-Gaussian distribution model of SWT wavelet coefficients [8]. Cui et al. proposes a low complexity SWT despeckling method based on LMMSE Wavelet Shrinkage[9]. 
Recently, the data-adapted kernel regression methods are widely used for restoration [10], which relies on not only the sample location and density, but also on the radiometric properties of these samples. Inspired by the basic idea of the data-adapted kernel regression methods, this paper modifies the parameter estimation method in [9] by the bilateral kernel regression to improve this despeckling method based on LMMSE Wavelet Shrinkage.

\section{SPECKLE MODEL}

Speckle noise in SAR images arises as a consequence of the coherent illumination used by radar. Within each ground resolution cell a large number of elementary reflectors reflects the radar wave towards the sensor. For a surface that is rough on the scale of the radar wavelength, the number of elementary reflectors is large enough to ensure the statistical independence in phase and amplitude of the elementary backscattered waves. For this type of areas, the speckle is fully developed. In this paper, the speckle is assumed to be fully developed, which is valid for homogeneous targets and weakly textured areas, but is only an approximation for point targets and extremely heterogeneous areas.

For a SAR image in which the speckle is fully developed, a multiplicative noise model is often employed, which can be expressed as

$$
v=f z
$$

where $v$ is the observed noisy signal, $f$ is the noise-free signal and $z$ is the normalized speckle random variable independent of $f$ with unit mean, respectively. The intensity image is considered in this paper, which is usually used one of SAR images formats. In the intensity format, $z$ follows the Gamma distribution. The multiplicative noise model in (1) can easily be decomposed into an additive model in the following form

$$
v=f z=f+f(z-1)=f+f z^{\prime}=f+b
$$

where $z^{\prime}$ is a random variable independent of $f$ with zero mean, and $b$ is the additive noise depending on the underlying unknown signal $f$.

\section{LMMSE DESPECKLING IN THE STATIONARY WAVELET DOMAIN}

In signal processing, the representation of signal plays a fundamental role. Wavelet transform is a powerful tool to facilitate the representation and analysis of transient signals. In order to preserve the translation invariance property, the stationary wavelet transform is introduced in this paper. It enables a better despeckling quality. A common despeckling procedure with wavelets is: 1) Compute the wavelet transform; 2) Remove speckle noise from the wavelet coefficients and 3) Reconstruct the despeckled image. The scaling coefficients are usually kept unchanged. During this procedure, the second step is the most important. In this paper, LMMSE technique is used to estimate the desired noise-free wavelet coefficient in the second step just H. Xie, L.E. Pierce, F.T. Ulaby, as [9].
Due to linearity of the wavelet transform, the additive model (2) remains additive in the transform domain as well

$$
w=y+n
$$

where $w$ is the observed wavelet coefficient, $y$ is the noisefree coefficient, $n$ is the additive noise. The LMMSE estimate of $y$ is derived as

$$
\hat{y}=\eta w
$$

where

$$
\eta=\frac{E\left[w^{2} \mid-E[w n]\right.}{E\left[w^{2}\right]}
$$

According to (4) and (5), it is necessary to estimate $E\left[w^{2}\right]$ and $E[w n]$ to calculate $\hat{y}$.

According to [9],

$$
\begin{aligned}
& E\left[w_{j}^{\mathrm{HL}}(a, b) n_{j}^{\mathrm{HL}}(a, b)\right] \\
& =\sigma_{Z}^{2} \sum_{l, k} h_{j}^{e q}(l)^{2} g_{j}^{e q}(k)^{2}\{E[v(a+l, b+k)]\}^{2},
\end{aligned}
$$

$$
\begin{aligned}
& E\left[w_{j}^{\mathrm{LH}}(a, b) n_{j}^{\mathrm{LH}}(a, b)\right] \\
= & \sigma_{z}^{2} \sum_{l, k} g_{j}^{e q}(k)^{2} h_{j}^{e q}(l)^{2}\{E[v(a+k, b+l)]\}^{2}, \\
& E\left[w_{j}^{\mathrm{HH}}(a, b) n_{j}^{\mathrm{HH}}(a, b)\right] \\
= & \sigma_{z}^{2} \sum_{l, k} g_{j}^{e q}(l)^{2} g_{j}^{e q}(k)^{2}\{E[v(a+l, b+k)]\}^{2},
\end{aligned}
$$

where HL, LH and HH stand for the orientation subbands corresponding to the horizontal, vertical and diagonal directions respectively. $\sigma_{z}^{2}$ is the variance of $\mathrm{z}$ and $\sigma_{z}^{2}=1 / L$ in L-look image. $h_{j}^{e q}(l)$ and $g_{j}^{e q}(k)$ are the coefficients of the equivalent filters at the scale $2^{j}$.

$$
\begin{aligned}
& h_{j}^{e q}(l)=h_{j-1}\left(m_{j}\right) h_{j-2}\left(m_{j-1}\right) \Lambda h_{0}\left(m_{1}\right), \\
& g_{j}^{e q}(k)=g_{j-1}\left(n_{j}\right) h_{j-2}\left(n_{j-1}\right) \Lambda h_{0}\left(n_{1}\right),
\end{aligned}
$$

where $l=m_{1}+m_{2}+\Lambda+m_{j} \quad, \quad k=n_{1}+n_{2}+\Lambda+n_{j}$, $h_{j-1}\left(m_{j}\right)$ is the coefficient of the lowpass filter at the scale $2^{j}$, and $g_{j-1}\left(n_{j}\right)$ is the coefficient of the highpass filter at the scale $2^{j}$. 
If the observed noisy signals are assumed to be identically distributed within the neighborhood system of pixel $(a, b)$, its mean at pixel $(a, b)$ can be estimated by

$$
E[v(a, b)]=\frac{1}{K \times K} \sum_{(l, k) \in \kappa(a, b)} v(l, k),
$$

where $\kappa(a, b)$ denotes the neighborhood system of pixel $(a, b)$ and $K \times K$ is corresponding dimension.

In [9], $E\left[w^{2}\right\rfloor$, which is the second-order moment of the wavelet coefficient at pixel $(a, b)$, is estimated using neighboring coefficients in the following form by assumed that theirs distribution is identical in the neighborhood system of pixel $(a, b)$

$$
E\left[w(a, b)^{2}\right]=\frac{1}{M \times M} \sum_{(l, k) \in v(a, b)} w(l, k)^{2},
$$

where $v(a, b)$ denotes the neighborhood system of pixel $(a, b)$ and $M \times M$ is corresponding dimension. However, the wavelet coefficients belong to one of three subbands, corresponding to three orientations in the image, and the coefficients carry horizontal, vertical, or diagonal information. Thus, significant coefficients form clusters with predominantly the horizontal, vertical, or diagonal direction, depending on the orientation subband. Therefore, the assumption that wavelet coefficient distribution is identical in the neighborhood system of pixel $(a, b)$ is not well adaptive to the prior knowledge about the spatial clustering of the wavelet coefficients. To overcome this shortcoming, the estimation method of the second-order moment of the wavelet coefficient is modified by incorporating the structure information into the estimation proccess using the bilateral kernel regression.

For the wavelet coefficient at pixel $(a, b)$, we use the similar neighboring pixels to estimate its variance:

$$
E\left[w(a, b)^{2}\right]=\sum_{(l, k) \in v(a, b)} \beta(l, k) w(l, k)^{2} .
$$

The weights can be calculated by

$$
\beta(l, k)=\frac{K[w(l, k), w(a, b)]}{\sum_{(l, k) \in v(a, b)} K[w(l, k), w(a, b)]},
$$

where

$$
\begin{aligned}
& K[w(l, k), w(a, b)] \\
& =\exp \left(\frac{-[w(l, k)-w(a, b)]^{2}}{h_{w}^{2}}\right)
\end{aligned}
$$

where $h_{w}$ is the smoothing scalar. The similarity of neighboring pixels is measured by the Gaussian kernel, which is the decreasing function of the difference between the adjacent coefficients. By the weight function, the similar wavelet coefficients with the reference are selected to estimate the variance in (13).

Once $E[w n]$ and $E\left[w^{2}\right]$ are calculated according to (6), (7), (8) and (13), y can be estimated in (4).

\section{EXPERIMENTAL RESULTS}

Performance assessment for SAR image despeckling is a difficult task for lack of original noiseless image. Therefore, experiments in this paper are carried out on simulated SAR images, which are optical images corrupted by simulated specke in intensity format. The performance of the proposed despecking algorithm for simulated SAR images will be illustrated with a quantitative and a qualitative performance measure. The signal-to-noise (SNR) is used as the quantitative measure. The qualitative measure is the visual quality of the resulting image.

The performance will be illustrated on the $256 \times 256$ test images: Lena and Peppers. The input image is decomposed over three levels with stationary wavelet transform. The variation on the CDF-(spline)-filters is used. Primal and dual wavelets have four vanishing moments. These wavelets are rather popular in image processing.

For comparison, the improvements in terms of SNR of the modified method, the low complexity SWT despeckling method based on LMMSE Wavelet Shrinkage(LCLMMSE) [9] and the two related state-ofthe-art methods: GMMMSE[8] and Kuan filter[1] are summarized in Table I, Table II respectively. All despeckling methods obviously achieve a higher gain in SNR when the input image is noisier. When input SNR is low, LCLMMSE performs better than the other methods. But with the improvement of input SNR, the proposed modified method achieves higher output SNR. This is mainly because with the reduction of the noise, the modified method can better select similar wavelet coefficients to estimate the parameter $E\left[w^{2}\right]$. When the precision of the parameter $E\left[w^{2}\right]$ is improved, the performance of denoising method is also improved.

Fig .1 presents the original Lena image and the same image with artificial speckle noise $(\mathrm{L}=200)$. The result of the proposed modified method and the other related methods for noisy Lena are shown in Fig .2. Fig .3 presents the original Peppers image and the same image with artificial speckle noise $(\mathrm{L}=100)$. The result of the proposed modified method and the other related methods for noisy Peppers are shown in Fig .4. They are consistent with quantitative results. The proposed method and LCLMMSE achieve the better noise reduction performances in homogeneous areas. Compared with the LCLMMSE, the proposed modified method provides better texture preservation performances.

TABLE I. COMPARISON OF QUANTITATIVE RESULT FOR LENA IMAGE IN SNR (DB)

\begin{tabular}{|l|c|c|c|c|c|c|}
\hline Looks & 40 & 100 & 150 & 200 & 300 & 500 \\
\hline Noise & 9.30 & 13.30 & 15.07 & 16.30 & 18.05 & 20.28 \\
\hline This Study & 16.18 & 19.03 & 20.48 & 21.38 & 22.54 & 24.13 \\
\hline LCLMMSE[9] & 16.74 & 19.22 & 20.42 & 21.25 & 22.41 & 23.92 \\
\hline GMMMSE[8] & 15.13 & 17.84 & 19.1223 & 20.02 & 21.22 & 22.87 \\
\hline Kuan[1] & 14.40 & 17.21 & 18.54 & 19.47 & 20.82 & 22.56 \\
\hline
\end{tabular}


TABLE II. COMPARISON OF QUANTITATIVE RESUlT FOR PEPPERS IMAGE IN SNR (DB)

\begin{tabular}{|l|c|c|c|c|c|c|}
\hline Looks & 20 & 40 & 100 & 200 & 300 & 500 \\
\hline Noise & 4.92 & 7.89 & 11.89 & 14.88 & 16.65 & 18.84 \\
\hline This Study & 13.79 & 15.71 & 18.35 & 20.37 & 21.50 & 22.94 \\
\hline LCLMMSE[9] & 14.11 & 15.89 & 18.28 & 20.21 & 21.29 & 22.71 \\
\hline GMMMSE[8] & 12.19 & 14.06 & 16.73 & 18.93 & 20.27 & 21.84 \\
\hline Kuan[1] & 11.66 & 13.54 & 16.25 & 18.41 & 19.68 & 21.33 \\
\hline
\end{tabular}
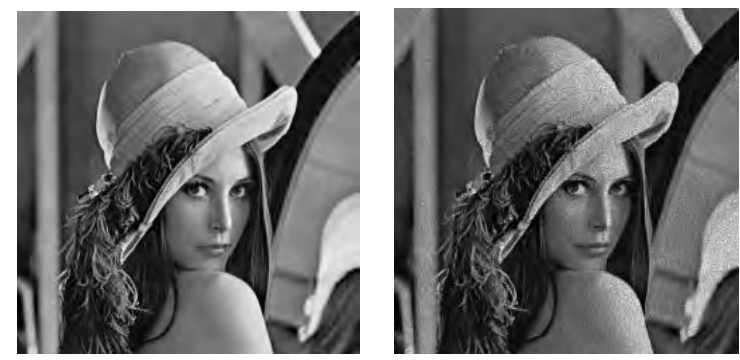

Figure 1. Original Lena image (left) and the same image with artificial speckle noise ( $L=200$, right).

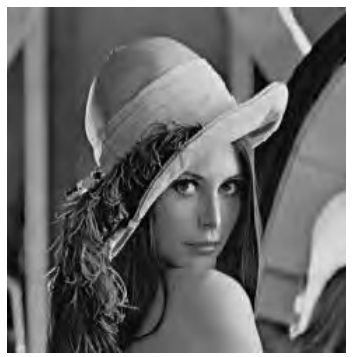

(a) The proposed method

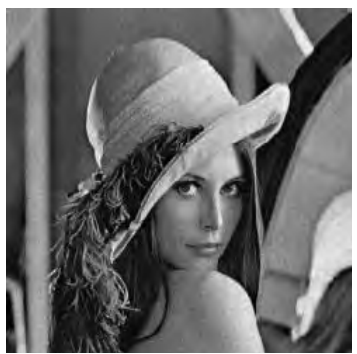

(c) GMMMSE

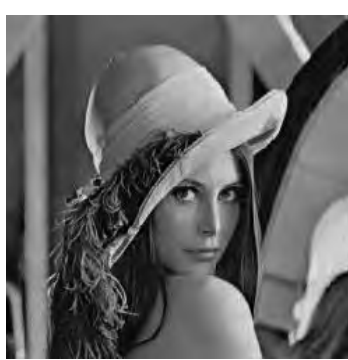

(b) LCLMMSE

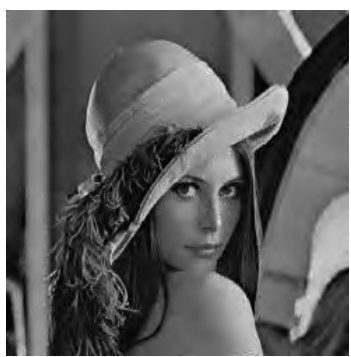

(d) Kuan
Figure 2. Comparison of dfferent despeckling methods for noisy Lena image of Fig .1
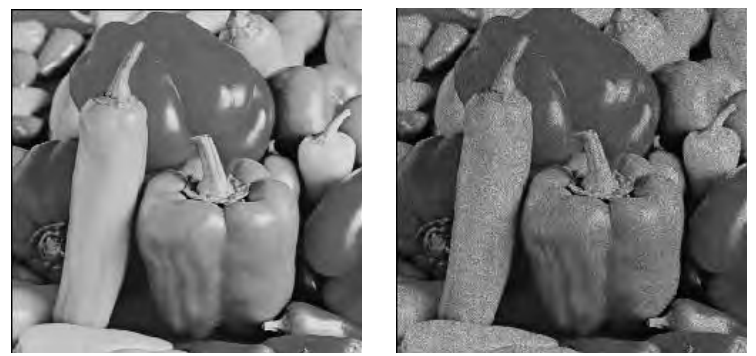

Figure 3. Original Peppers image (left) and the same image with artificial speckle noise ( $\mathrm{L}=100$, right).

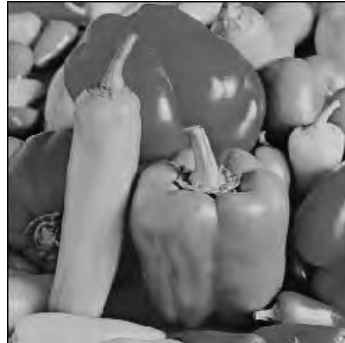

(a) The proposed method

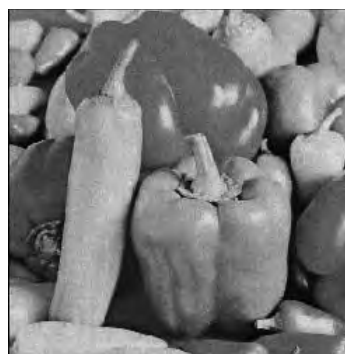

(c) GMMMSE

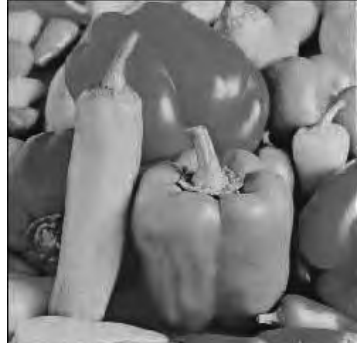

(b) LCLMMSE

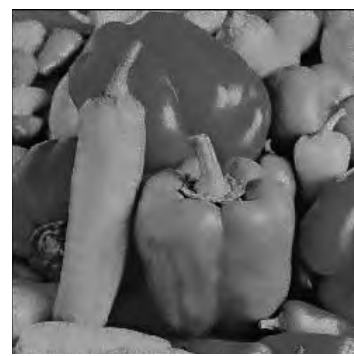

(d) Kuan
Figure 4. Comparison of dfferent despeckling methods for noisy Peppers image of Fig .3

\section{CONCLUSION}

To improve the despeckling performance, an adaptive method based on LMMSE Wavelet Shrinkage is proposed in this paper. This method is an modification of LCLMMSE filter in [9]. Based on the distribution property of wavelet coefficients, the parameter of LMMSE wavelet shrinkage is estimated by taking advantage of bilateral kernel regression. Experiment results demonstrate this method improves the despeckling performance quantitatively and qualitatively when the noise is not serious.

\section{ACKNOWLEDGMENT}

This work was supported by Fundamental Research Funds for the Central Universities (DC110324 and DC110309)

\section{REFERENCES}

[1] D.T. Kuan, A.A. Sawchuk, T.C. Strand, P. Chavel, "Adaptive restoration of images with speckle," IEEE Transactions on Pattern Analysis and Machine Intelligence, vol.7, 1985, pp. 165-177.

[2] S. Parrilli, M. Poderico, C.V. Angelino and L. Verdoliva, "A nonlocal SAR image denoising algorithm based on LLMMSE wavelet shrinkage," IEEE Transactions on Geoscience and Remote Sensing, vol.50, 2012, pp. 606-616.

[3] H. Xie, L.E. Pierce, F.T. Ulaby, "SAR speckle reduction using wavelet denoising and Markov random field modeling," IEEE Transactions on Geoscience and Remote Sensing, vol.40, 2002, pp. 2196-2212.

[4] S.G. Chang, B. Yu, M. Vetterli, "Adaptive wavelet thresholding for image denoising and compression," IEEE Transactions on Image Processing, vol.9, 2000, pp.1532-1546.

[5] H. Xie, L.E. Pierce, F.T. Ulaby, "Despeckling SAR images using a low-complexity wavelet denoising process," Proceedings of International Geoscience and Remote Sensing Symposium, 2002, pp. 321-324.

[6] S. Foucher, G.B. Bénié, J.M. Boucher, "Multiscale MAP filtering of SAR images," IEEE Transactions on Image Processing, vol.10, 2001, pp.49-60. 
[7] F. Argenti, L. Alparone, "Speckle removal from SAR images in the undecimated wavelet domain," IEEE Transactions on Geoscience and Remote Sensing, vol.40, 2002, pp.2363-2374.

[8] M. Dai, C. Peng, A.K. Chan, D. Loguinov, "Bayesian wavelet shrinkage with edge detection for SAR image despeckling," IEEE Transactions on Geoscience and Remote Sensing, vol. 42, 2004, pp. 1642-1648.
[9] Y.Q. Cui, T. Zhang, S. Xu, W.M. Yu. "Image despeckling based on LMMSE wavelet shrinkage," PRZEGLĄD ELEKTROTECHNICZNY, vol. 88, 2012, pp. 269-272.

[10] H. Takeda, S. Farsiu and P. Milanfar, "Kernel Regression for Image Processing and Reconstruction," IEEE Transactions on Image Processing, vol.16, 2007, pp.349-366. 